\title{
Selection of Conductive Composite Through Weighed Normalized Fuzzy Method in Order to be used in Electrostatics Filters Absorbing Plates
}

\author{
Ali Akbar Lotfi NEYESTANAK, Gasem ASADI, Saeed DANESHMAND
}

\begin{abstract}
In this paper, due to some problems deriving from using multiple inputs in fuzzy logic procedure, a simpler high demanding method, which can eliminate some complicated fuzzy membership functions trends is represented. This method is so-called weighed normalized fuzzy method. Despite simple calculations used in the method and user capability to use fuzzy membership functions, ranking utilized in this method is as authentic as other conventional fuzzy logic procedures. Regarding suitable properties of composites which can solve problems happening due to intrinsic characteristic of materials used in electrostatic precipitators, in this investigation wide spectrum of resins and fibres used in manufacturing conductive composites is studied. In addition, some of them are selected through material selection methods with regard to their attributes and high conductivity property, which is suitable for absorbing plates. Selecting best conductive composites among considered conductive composites through this novel method is the final aim of this paper. The results show that vinyl ester, which belongs to Vinyl Ester Resin Woven Carbon and Glass (VRWCG) series, has the most beneficial specification in comparison with other thermoset resins for this intended application. Moreover, vinyl ester resins show better stability in acidic electro filter media.
\end{abstract}

Keywords: electrostatic precipitator; fuzzy logic method; material selection; vinyl ester conductive composite

\section{INTRODUCTION}

Material selection is the most important step in designing electrostatic precipitator's constructions. So utilizing material selection methods invented through past decades is so beneficial to this purpose. Multiple methods like Digital Logic (DL), Modified Digital Logic (MDL), $\mathrm{Z}$ transfer and fuzzy logic methods have received attention in recent years. Considering merits of each method for material selection issue, they suffer from some demerits too. Based on inherent limitations of these methods, it is highly mandatory to introduce a novel method which resolves such deficiencies, with having less complexity. Because of multiple inputs used in the fuzzy logic method, it has some problems which should be resolved. Therefore, it is better to use a simpler and high demanding method that eliminates complicated fuzzy membership functions. It is so-called weighed normalized fuzzy method. Despite simple calculations used in the method and user capability to use fuzzy membership functions, ranking utilized in this method is as valid as other conventional fuzzy logic procedures. Material selection methods are usually used for manufacturing a new part; however, it is also useful for some other purposes like material substitution. In order to select or substitute materials for absorbing plates of electrostatic filters, conventional material selection methods are used to choose among all suitable conductive polymeric composite materials regarding their attributes. It is of high importance to select some specific materials that satisfy all limitations of electro filtering systems while maintaining their performance and efficiency. After designing step, it is focused on comparing properties of some alternative materials and selecting the best one which has the most conformity with requirements needed by this equipment. It is so-called material selection. As shown in Fig. 1, in order for substituting materials in decision-making step, a variety of design steps and corresponding activities for material selection and manufacturing processes are considered. As opposed to scientific facts that there is usually one answer for a given problem, there are some different possible results on the ground that a large variety of advantages, limitations, and essentials of given methods should be considered for material substituting and decision-making step. It is worthy to mention that products with the same application are usually manufactured by various manufacturers through different processes. Considering material role in designing step and various kinds of available materials, material selection is of high importance. There are various material selection methods with different strengths and weaknesses. Subdivisions of methods are shown in figure 2. Regarding inherited problems of these methods, there is an attempt to introduce a novel method which not only alleviates existing deficiencies and lowering complications but also promotes result accuracy. In general, based on qualitative and quantitative properties of materials, several methods have been presented. Ashby et al [1] have reviewed various strategies and methods of material selection. Based on them, material selection is categorized in three groups. (a) Free searching based on quantitative analyses; the most popular one in this category is graphic or ranking method [2]. (b) Based on checklist or questionnaire and experimental method like reports [3-6] which are based on science, intelligent data, or [7] which is question based structure, resulting in an optimal design solution. (c) Inductive reasoning type or analogous procedure.

As material selection processes can be considered as a decision-making problem, neural network; A type of artificial intelligence [8] or fuzzy logic approaches [9, 10] are employed to qualitatively evaluate material properties. Furthermore, multi criteria fuzzy logic methods [11] or ranking techniques using similar ideal solutions are presented by other investigators [12]. Moreover, peripheral [13] or environmental costs [14], economic or technology oriented materials should be seen in the decision-making process. Simultaneously, a large number of quantitatively material selection methods are developed in order to analyse a huge mass of data involved in the material selection process so that a systematic evaluation will be arranged [15]. For example, Ashby [16, 17] has introduced material selection flowcharts. He has proposed Multi-objective optimization methods for mutual aspects of decision-making [18]. Gorton et al. [19] has used fatigue specifications charts to design minimal weight 
based on infinite life span. Another aspect for material selection issues is properties weighing method (WPM). In this method, alternative materials are being ranked based on their performance indices through simple mathematical calculations [20]. In some cases, where so many material properties are involved and there is no priority in their importance, determining weighting factor $(\alpha)$ will be so complicated. Therefore, the reliability of material selection method falls down intensively. As a result, digital logic (DL) method has been presented to determine $\alpha$. This method was further modified by Menshadi et al. [21] because of some problems and deficiencies, existing in a normalizing process, this method is named MDL (modified digital logic), involving the nonlinear normalizing function with some changes in conventional digital logic method, yet it has some deficiencies in normalizing function, which is finally corrected by Fayazbakhsh et al. [22, 23] using normalizing function $\mathrm{Z}$ in a statistical way. This method is called Ztransformation method.

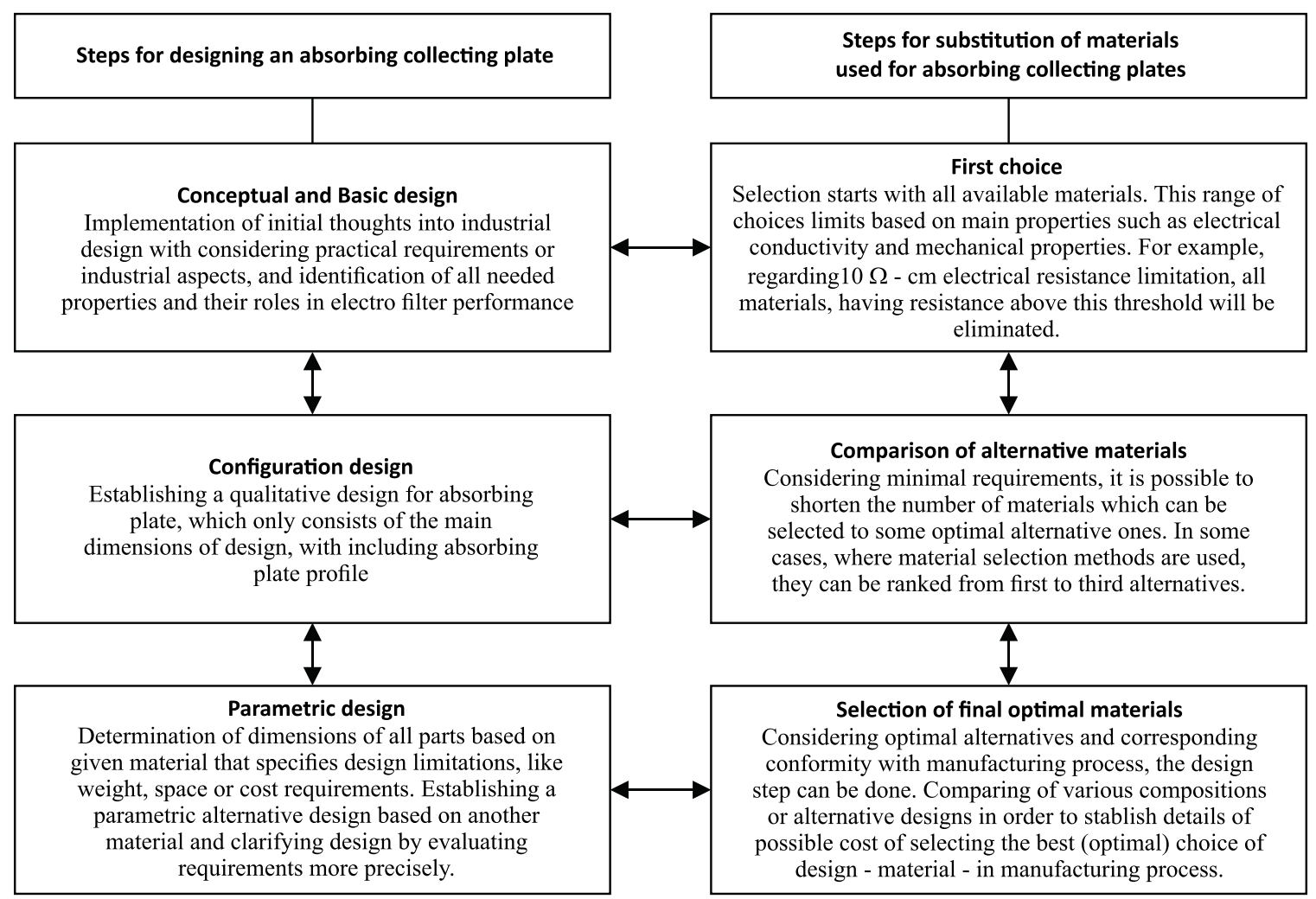

Figure 1 Substitution steps of absorbing plate materials

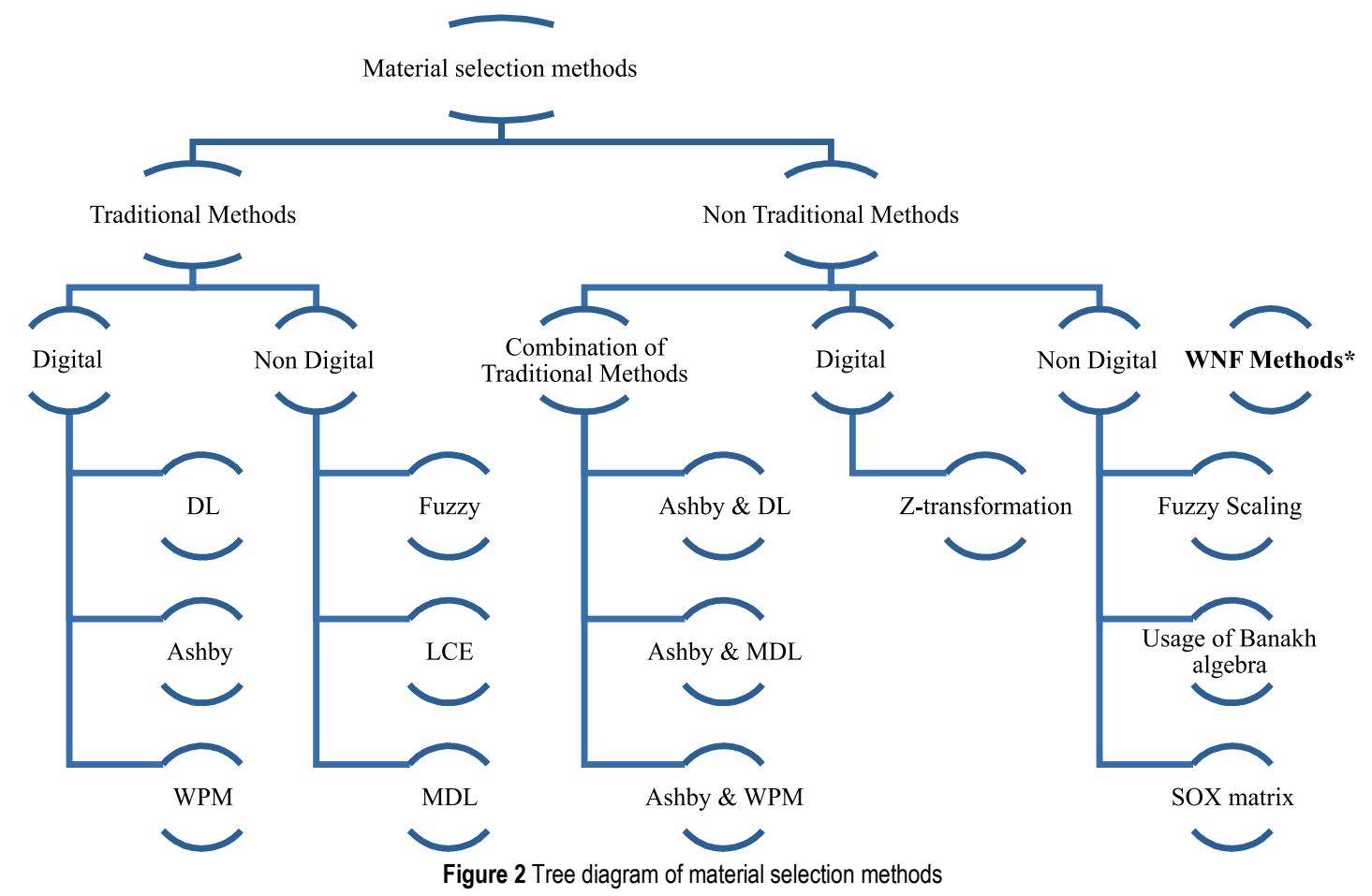




\section{SAMPLE PREPARATION}

The hybrid nanocomposites of this study were fabricated as seen in Fig. 3. It consists of three fundamental components: advanced fibre fabric (with high amount: $\sim 50 \%$ ), polymer matrix (phenolic or vinyl ester) and graphite nanopowder with different percentages. In this research, two types of fabrics were used. One type, named "Torayca", is made of carbon fibre, with having young's modulus (231 GPa), tensile strength $(4.53 \mathrm{GPa})$ and fracture strain $(1.5 \%)$, the other one, named "Hexcel", was built of glass fibre (S-glass), with having Young's modulus ( $86 \mathrm{GPa})$, tensile strength $(4.58 \mathrm{GPa})$ and fracture strain $(0.01 \%)$. These two types were used as the reinforcement material in phenolic resin conductive composites and vinyl ester resin conductive composites. The applied phenolic resin was a resol resin with a density of $1.21 \mathrm{gr} / \mathrm{cm}^{3}$ under commercial name "Resitan IL800", the Rositan company's product. And the applied epoxy vinyl ester was Hetron epoxy vinyl ester resins with a density of $1.04 \mathrm{gr} / \mathrm{cm}^{3}$ under commercial name "Hetron 922", the Ashland company's product. The applied graphite nanopowder was the Merck company's product with a density of $2.2 \mathrm{gr} / \mathrm{cm}^{3}$ (at $20{ }^{\circ} \mathrm{C}$ ) and the particle size less than $70 \mu \mathrm{m}$ and typical particle thickness of $0.5 \mu \mathrm{m}$. cobalt naphthalene and $\mathrm{N}-\mathrm{N}$ dimethyl aniline were utilized as the catalyst, accelerator, and promoter, respectively. Four constructed composite families investigated in this study are: (1) four layer hybrid resolve phenol composites reinforced with different density of graphite nanopowder and carbon fabric (abbreviated PRWC) as seen in Fig. 3a, (2) four layer hybrid resol phenol composites reinforced with different density of graphite nanopowder and carbon fabric in two external layers and glass fabric in two inner layers (abbreviated PRWCG) as seen in Fig. 3b, (3) four layer hybrid Hetron epoxy vinyl composites reinforced with different percentages of graphite nanopowder and carbon fabric (abbreviated VRWC) as seen in Fig. 3a, (4) four layer hybrid Hetron epoxy vinyl ester composites reinforced with different percentages of graphite nanopowder and carbon fabric in two external layers and glass fabric in two inner layers (abbreviated VRWCG) as seen in Fig. $3 \mathrm{~b}$, all of these four types were constructed as a plate with $200 \mathrm{~mm}$ length, $20 \mathrm{~mm}$ width and $1.5 \mathrm{~mm}$ thickness.

For this purpose, resol phenol or epoxy vinyl ester was completely mixed with the different amount of graphite nanopowder $(5 \%, 10 \%, 15 \%$ and $20 \%)$ by means of a Brabender P12200 blender with a velocity of $60 \mathrm{rmp}$ for $30 \mathrm{~min}$. and finally, accelerator, promoter, and catalyst (each of them 1\%) were added to the mixture. Then, by use of hand layup method glass and carbon fabrics with similar amount of fibre were soaked in the blended resin and moulded. At the end, for resolve phenol, thermal press curing was done after moulding at 200 bar pressure and $160{ }^{\circ} \mathrm{C}$ temperature for 10 minutes. And for epoxy vinyl ester, thermal press curing was done after moulding at 200 bar pressure and $120{ }^{\circ} \mathrm{C}$ temperature for 20 minutes.

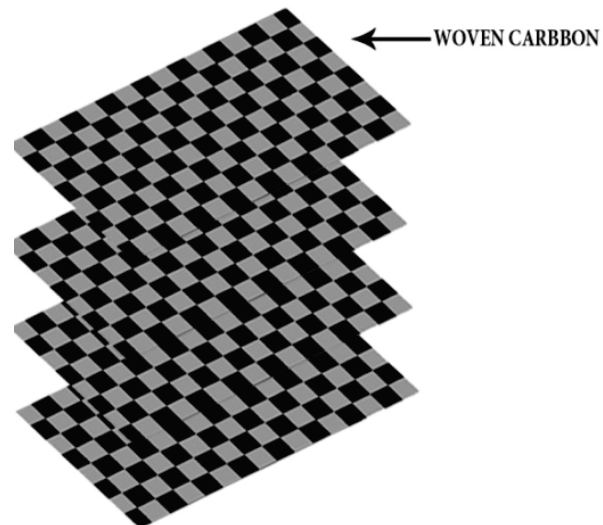

(a) Woven woven

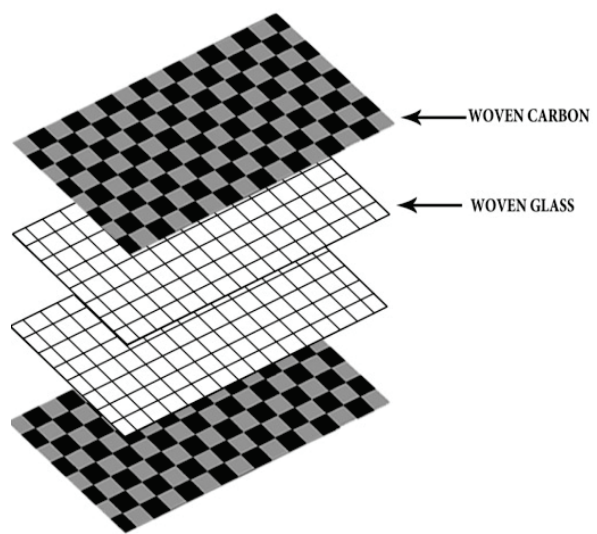

(b) Glass and Carbon woven

Figure 3 Schematic figure of woven reinforced polymers composite hybrid with nanopowder graphite

\section{WEIGHED METHOD MATERIAL SELECTION}

In weighed method, a certain weight ratio, which depends on the performance of a piece of material as it is in service, is assigned to each material's properties. The weight of material properties is acquired by multiplying the value of each property in the weight factor $(\alpha)$. Unique values of each substance's properties are used to gain weight ratio relative performance index material $(\gamma)$. Higher performance materials are suitable for use [20].

Weight factor of any purpose is obtained by dividing the number of positive decisions of each target $(\mathrm{m})$ by the total number of potential decisions $(N)$. In this case $\sum \alpha=1$ and Materials performance index can be calculating by calculating by $\gamma=\sum_{i=1}^{n} \beta_{i} \alpha_{i}$.

Weighed method has difficulties when we combine some properties. This may cause illogical results, especially when the mechanical, physical and chemical properties are combined with a wide range of different numerical values. Therefore, one of the properties at higher levels, and may even find more influence with regard to the weight factor, will be an unpleasant combination of these forms has been fixed by introducing scaling factors.

\section{WEIGHED NORMALIZED FUZZY METHOD MATERIAL SELECTION}

The conventional fuzzy method is a complicated method that needs a great deal of experience to be used efficiently. It is so time-consuming owing to the fact that 
the user should write corresponding "if - then" programs for every decision. This results in more complexity in creating space fuzzy when there are more than two criteria for each decision making. Further, numerous rules are needed to make a decision space which includes all required aspects. For example when there are 5 decision criteria for a given problem and every criterion has 3 possible states, there should be written $3^{\wedge} 5$ "if - then" programs to include all possible states, so it is not a suitable case for material selection issues, which are usually involved with more than two criteria. In addition, selecting of some of these rules for a decision-making process requires good experience in fuzzy logic. Another problem which can be seen in this method is that there is no preference or importance ranking among considered criteria. Although some criteria may be more important than others, there is no preference or special definition for them in this method. Moreover, it depends on the number of rules; therefore, adding or reducing "if - then" rules, can change the output. The novel method, presented here, tries to eliminate "if - then" rules and use fuzzy normalizing, which is much easier than before. The importance of switching to this method can be noticed when there are more than two criteria for a given problem. In the presented method (like "if - then" rules) it is possible to select membership function freely. It means that when design limitations are determined, the designer can arbitrarily use these limitations to determine membership coefficients. Also, where it is too complex to determine them, the designer can follow previous methods and utilize minimal and maximal levels of specifications in order to determine these functions. In this method, the normalizing function is designed in conformity with membership function form and capabilities. Therefore, as opposed to some other methods like surface torque method that output membership function's form is not of enough importance in antifuzzing, there is so much attention paid to output membership functions in this method. It is simple to program and so practical for problems like material selection case where there are a high number of inputs that should be considered. For problems which have less number of inputs, when "if - then" rules are selected and written properly, fuzzy logic can probably be carried out more quickly using "if - then" rules, but as mentioned before because of high number of input materials in decision-making problem, the presented method is simpler and quicker than conventional methods. It is worthy to mention that in the light of Rao's method it is possible to determine membership function's equivalent values. In most of the papers presented by Rao, there is no notification about how to determine these values. Consequently, by trial and error method for membership function's geometry, it is possible to find how it works. It is worthy to note that normalizing function of this method has a new idea. In order to use modified fuzzy method, some steps should be taken as follows:

Step 1: Listing all possible alternatives and forming material characteristics matrix. In the first step all possible composite alternatives, which are available for the designer to make a special design are listed, then some characteristics which are more substantial are selected.
There will be a matrix or special table composed of values related to those properties (Tab. 1).

Step 2: Definition of target function and membership functions for composites properties. In this step, according to a designer's opinion, bad, medium, good and excellent areas for every material's properties (composites) are given. The following curves depict those areas (Fig. 4).

Step 3: Determination of equivalent values according to Rao's method and following Fig. 5 equivalent values of medium state are calculated as Eq. (1).

$$
\begin{aligned}
& U_{D_{O M}}=\frac{1+D_{L i}-D_{R i}}{2}= \\
& =\frac{1}{2} \cdot\left[\frac{\left(x_{3 i}-x_{\min }\right)}{\left(\left(x_{\max }-x_{\min }\right)-\left(x_{2 i}-x_{3 i}\right)\right)}+1-\frac{\left(x_{\max }-x_{1 i}\right)}{\left(\left(x_{\max }-x_{\min }\right)+\left(x_{2 i}-x_{1 i}\right)\right)}\right] \\
& i=1,2, \ldots, n
\end{aligned}
$$

Considering Fig. 5 and Eq. (1) $x_{1}, x_{2}, x_{3}, x_{\min }, x_{\max }$ are points related to every composite's properties and will be the inputs in the code written in Matlab software. Considering suitable values from Eq. (1), the value of an equivalent function, $U$, can be calculated.

Step 4: Entering properties of alternative composites. In this step, composite properties are converted to a special matrix which will be entered into the code (or program) written in matlab environment.

Step 5: Normalizing the input values. Given that matrix $\boldsymbol{D}$ is "input values matrix", according to the Eq. (2) it is necessary to normalize input values. Sufficient attention needs to be paid in determining function $D O M_{i}$, $D O M_{i+1}$ properly. There is a special section in the code for calculating mentioned functions.

$$
\begin{aligned}
& \boldsymbol{D}=\left[\begin{array}{llllll}
x_{1} & x_{2} & \ldots & x_{j} & \ldots & x_{m}
\end{array}\right] \\
& r_{j}=\frac{1}{2} \cdot\left[\begin{array}{l}
\frac{\left(D^{\prime} M_{i+1}\left(x_{3 j}\right)-D_{O M}\left(x_{3 j}\right)\right) \cdot\left(U_{D O M}+1-U_{D O M i}\right)}{\left(1-\min \left(D_{D M}\left(x_{3 j}\right), D O M_{i}\left(x_{3 j}\right)\right)\right)}+ \\
+\left(U_{D O M i+1}+U_{D O M i}\right)
\end{array}\right] \\
& x_{1 j} \leq x_{3 j} \leq x_{1 j+1} \\
& \boldsymbol{R}=\left[\begin{array}{llllll}
r_{1} & r_{2} & \ldots & r_{j} & \ldots & r_{m}
\end{array}\right]
\end{aligned}
$$

Step 6: Determination of weight coefficients. In this step, according to designer's will, weights coefficients (fraction of 1) are given to each property.

Step 7: Determination of the normal matrix. According to Eqs. (5) and (6), normal matrix will be made.

$v_{j}=w_{j} \cdot r_{j}$
$\boldsymbol{V}=\left[\begin{array}{llllll}v_{1} & v_{2} & \ldots & v_{j} & \ldots & v_{m}\end{array}\right]$

Step 8: Determination of fuzzy output. In this step, using the Eq. (7) and adding normalized output values that are virtually composite's fuzzy score, the fuzzy output will be determined.

$$
C^{+}=\sum_{j=1}^{m} v_{j}
$$


In this special case, initial output for alternative materials' (composites) ranking is as in the Tab. 2 .

It may be assumed that the procedure is completed at this step, but the final result will be clarified when output fuzzy result becomes anti-fuzzed on index material function whose bad, medium, good and excellent areas are determined previously by the designer.

Step 9: Anti fuzzing the output. According to Eq. (8), anti-fuzzing is done by shaping a given equation for every portion of the membership functions curve (Fig. 1).

$$
\begin{aligned}
& \int\left[\begin{array}{l}
\frac{\left(D_{O M M_{i+1}}(x)-D_{O M}(x)\right) \cdot\left(D_{D O M i+1}-D_{D O M i}\right)}{\left(1-D O M_{i}(x)\right)} \\
+\left(D_{D O M i+1}+D_{D O M i}\right)
\end{array}\right] \cdot \frac{1}{2}=x_{3}{ }^{+}, \\
& x_{3}{ }^{+} \geq \frac{D_{D O M i+1}+D_{D O M i}}{2} \\
& {\left[\begin{array}{l}
\left.\frac{\left(D O M_{i+1}(x)-D_{i}(x)\right) \cdot\left(D_{D O M i+1}-D_{D O M i}\right)}{\left(1-D O M_{i+1}\right.}(x)\right) \\
+\left(D_{D O M i+1}+D_{D O M i}\right)
\end{array}\right] \cdot \frac{1}{2}=x_{3}{ }^{+},} \\
& x_{3}{ }^{+} \leq \frac{D_{D O M i+1}+D_{D O M i}}{2}
\end{aligned}
$$

\begin{tabular}{|c|c|c|c|c|c|c|c|c|c|c|c|c|c|}
\hline Material & 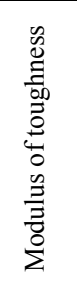 & 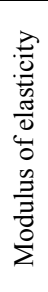 & 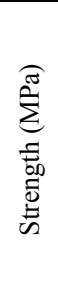 & 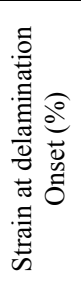 & 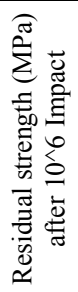 & 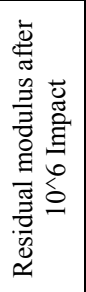 & 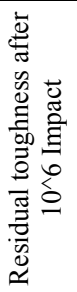 & 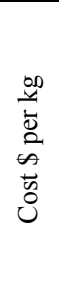 & 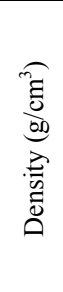 & 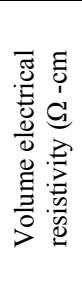 & 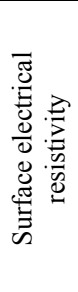 & 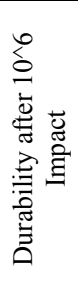 & 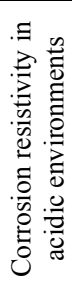 \\
\hline VRWCG & 794 & 29 & 282 & 0.61 & 83 & 11 & 404 & 343 & 1.71 & 5.197 & 2.980 & 70 & 98 \\
\hline VRWCG (5\% Nanopowder graphite) & 574 & 31 & 215 & 0.65 & 86 & 11 & 304 & 344 & 1.74 & 1.331 & 0.272 & 70 & 98 \\
\hline VRWCG (10\% Nanopowder graphite) & 746 & 30 & 198 & 0.65 & 111 & 13 & 416 & 344 & 1.76 & 0.947 & 0.177 & 70 & 98 \\
\hline VRWCG (15\% Nanopowder graphite) & 750 & 31 & 196 & 0.59 & 112 & 14 & 424 & 345 & 1.79 & 0.522 & 0.057 & 70 & 98 \\
\hline VRWCG (20\% Nanopowder graphite) & 677 & 32 & 208 & 0.67 & 123 & 16 & 461 & 345 & 1.82 & 0.522 & 0.057 & 70 & 98 \\
\hline VRWC & 529 & 70 & 251 & 0.39 & 149 & 22 & 375 & 606 & 1.32 & 1.536 & 0.245 & 70 & 98 \\
\hline VRWC (5\% Nanopowder Graphite) & 333 & 50 & 210 & 0.40 & 138 & 21 & 401 & 606 & 1.35 & 0.233 & 0.087 & 70 & 98 \\
\hline VRWC (10\% Nanopowder graphite) & 404 & 48 & 198 & 0.41 & 98 & 20 & 262 & 607 & 1.38 & 0.553 & 0.045 & 70 & 98 \\
\hline VRWC(15\% Nanopowder graphite) & 471 & 39 & 171 & 0.44 & 103 & 14 & 306 & 607 & 1.41 & 0.159 & 0.083 & 70 & 98 \\
\hline PRWCG & 417 & 30 & 139 & 0.48 & 71 & 5 & 329 & 327 & 1.82 & 0.070 & 0.095 & 50 & 80 \\
\hline PRWCG (5\% Nanopowder graphite) & 612 & 27 & 193 & 0.66 & 92 & 11 & 620 & 330 & 1.83 & 0.058 & 0.029 & 50 & 80 \\
\hline PRWCG (10\% Nanopowder graphite) & 908 & 25 & 173 & 0.70 & 83 & 9 & 361 & 333 & 1.84 & 0.061 & 0.016 & 50 & 80 \\
\hline PRWCG (20\% Nanopowder graphite) & 866 & 38 & 238 & 0.70 & 111 & 9 & 413 & 340 & 1.86 & 0.060 & 0.027 & 50 & 80 \\
\hline PRWC & 403 & 30 & 153 & 0.49 & 80 & 10 & 417 & 589 & 1.43 & 0.101 & 0.093 & 50 & 80 \\
\hline PRWC (5\% Nanopowder graphite) & 494 & 37 & 186 & 0.51 & 88 & 13 & 338 & 593 & 1.44 & 0.093 & 0.066 & 50 & 80 \\
\hline PRWC (10\% Nanopowder graphite) & 337 & 56 & 200 & 0.40 & 95 & 14 & 448 & 596 & 1.45 & 0.087 & 0.059 & 50 & 80 \\
\hline PRWC (15\% Nanopowder graphite) & 454 & 46 & 203 & 0.46 & 111 & 9 & 413 & 599 & 1.46 & 0.061 & 0.050 & 50 & 80 \\
\hline PRWC (20\% Nanopowder graphite) & 1054 & 60 & 212 & 0.58 & 82 & 8 & 440 & 602 & 1.47 & 0.058 & 0.043 & 50 & 80 \\
\hline
\end{tabular}

Table 1 List of all possible alternatives material specification
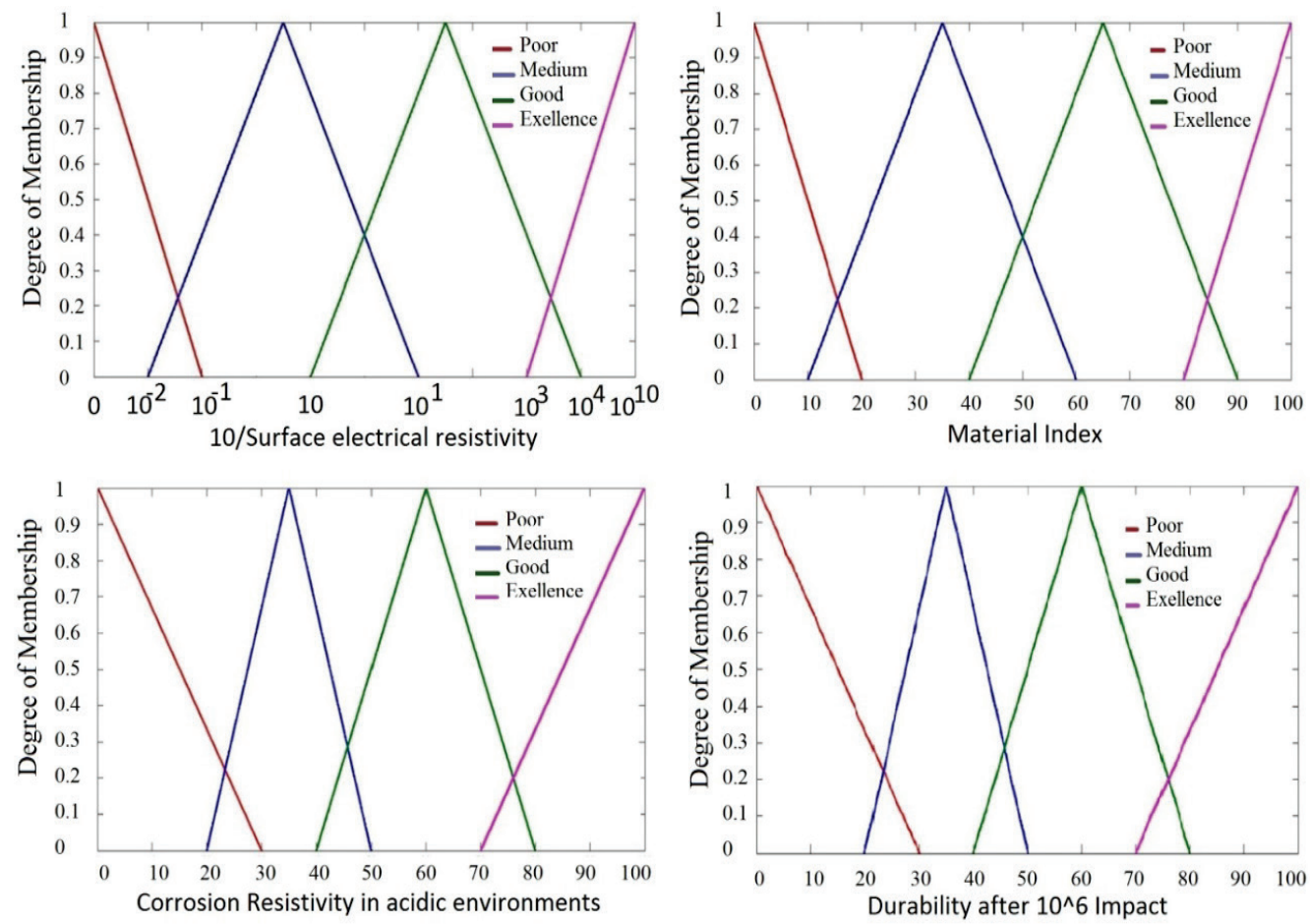

Figure 4 Bad-medium-good and excellent areas for some material attribute (composites) 
We should be cautious at this step. It is needed to subdivide the curve to reasonable portions to which different equations are assigned. Calculating $\operatorname{DOM}(x)$, we will be able to obtain $\mathrm{x}$ which is the final score of a given composite. In this step, final score or material index of the composites will be calculated.

Table 2 Initial output for alternative materials

\begin{tabular}{|l|c|c|}
\hline \multicolumn{1}{|c|}{ Material } & Fuzzy output & Rank \\
\hline VRWC & 0.3836 & 4 \\
\hline VRWC (5\% Nanopowder graphite) & 0.3737 & 7 \\
\hline VRWC (10\% Nanopowder graphite) & 0.3789 & 6 \\
\hline VRWC (15\% Nanopowder graphite) & 0.3817 & 5 \\
\hline VRWCG & 0.426 & 1 \\
\hline VRWCG (5\% Nanopowder graphite) & 0.3698 & 8 \\
\hline VRWCG (10\% Nanopowder graphite) & 0.3616 & 10 \\
\hline VRWCG (15\% Nanopowder graphite) & 0.3568 & 12 \\
\hline VRWCG (20\% Nanopowder graphite) & 0.3864 & 3 \\
\hline PRWC & 0.3337 & 18 \\
\hline PRWC (5\% Nanopowder graphite) & 0.3578 & 11 \\
\hline PRWC (10\% Nanopowder graphite) & 0.3472 & 15 \\
\hline PRWC (15\% Nanopowder graphite) & 0.3492 & 13 \\
\hline PRWC (20\% Nanopowder graphite) & 0.3953 & 2 \\
\hline PRWCG & 0.3366 & 17 \\
\hline PRWCG (5\% Nanopowder graphite) & 0.3478 & 14 \\
\hline PRWCG (10\% Nanopowder graphite) & 0.344 & 16 \\
\hline PRWCG (20\% Nanopowder graphite) & 0.3638 & 9 \\
\hline
\end{tabular}

Step 10: Comparison of conventional weighing with WNF procedure. In the case that scoring is done by conventional weighing procedure, there is a chance to compare the results with WFN. (Tab. 3)

To further validate the WNF technique, the conventional weighing procedure is also used in this case (The results of the comparison of material selection between these two methods are shown in Fig. 6. Three composites placed at the end of the list in Tab. 3 (in rows $21,22,23)$ are ranked as the first, second and third alternatives respectively. Because of getting an excellent score at most attributes they all get the same score in two procedures. In addition, the sixth composite has been placed in the fourth ranking in both procedures. The main difference between these two procedures is the $13^{\text {th }}$ composite that has got ranking $13^{\text {th }}$ in conventional procedure but $5^{\text {th }}$ in WNF procedure which shows designer's intervention has a direct impact on determining bad, medium, good and excellent areas. There are also other differences in the score of the other rows.

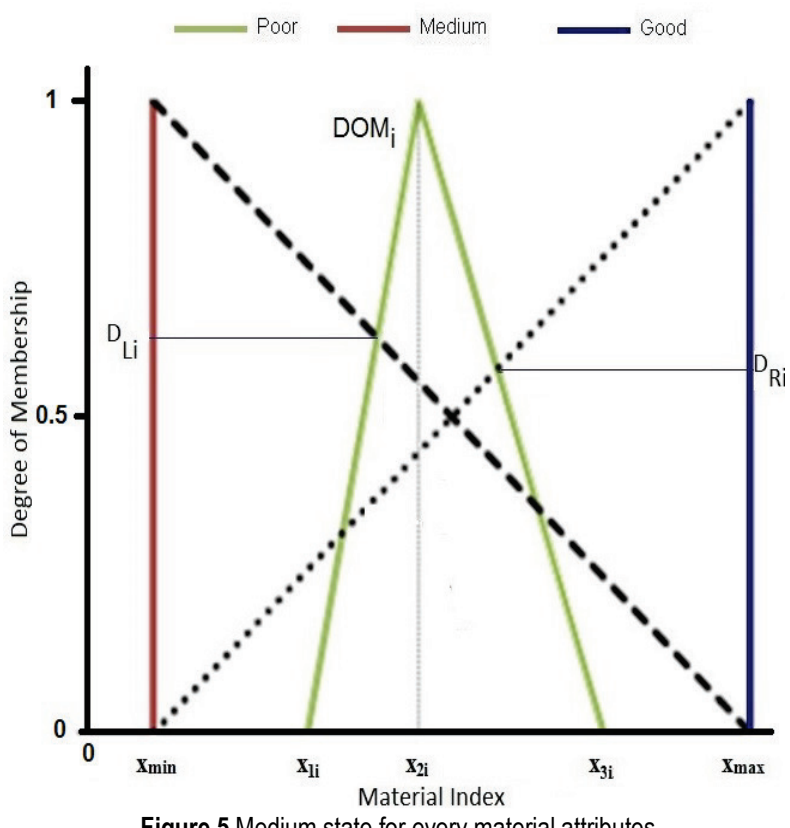

Figure $\mathbf{5}$ Medium state for every material attributes

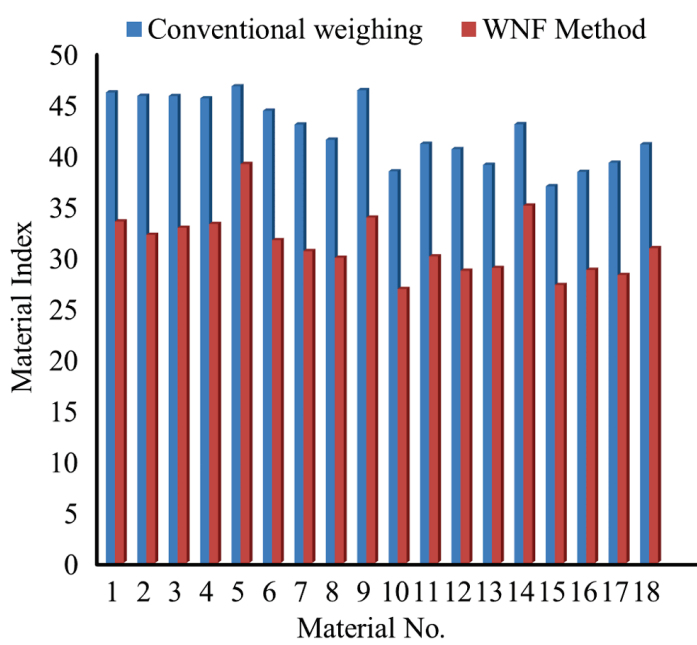

Figure 6 Comparing conventional weighing with WNF method (material no. versus material Index)

Table 3 Final score or material index of the composites

\begin{tabular}{|c|l|c|c|c|c|}
\hline \multirow{2}{*}{ No. Material } & & \multicolumn{2}{c|}{ WNF methods } & \multicolumn{2}{c|}{ Weight methods } \\
\cline { 3 - 6 } & & (material index) & Rank & (material index) & Rank \\
\hline 1 & VRWC & 33.477 & 4 & 46.122 & 3 \\
\hline 2 & VRWC (5\% Nanopowder graphite) & 32.166 & 7 & 45.785 & 4 \\
\hline 3 & VRWC (10\% Nanopowder graphite) & 32.857 & 6 & 45.771 & 5 \\
\hline 4 & VRWC (15\% Nanopowder graphite) & 33.229 & 5 & 45.541 & 6 \\
\hline 5 & VRWCG & 39.105 & 1 & 46.728 & 1 \\
\hline 6 & VRWCG (5\% Nanopowder graphite) & 31.644 & 8 & 44.330 & 7 \\
\hline 7 & VRWCG (10\% Nanopowder Graphite) & 30.567 & 10 & 42.976 & 9 \\
\hline 8 & VRWCG (15\% Nanopowder graphite) & 29.918 & 12 & 41.491 & 10 \\
\hline 9 & VRWCG (20\% Nanopowder graphite) & 33.857 & 3 & 46.341 & 2 \\
\hline 10 & PRWC & 26.857 & 18 & 38.390 & 16 \\
\hline 11 & PRWC (5\% Nanopowder graphite) & 30.052 & 11 & 41.085 & 11 \\
\hline 12 & PRWC (10\% Nanopowder graphite) & 28.645 & 15 & 40.566 & 13 \\
\hline 13 & PRWC (15\% Nanopowder graphite) & 28.915 & 13 & 39.023 & 15 \\
\hline 14 & PRWC (20\% Nanopowder graphite) & 35.030 & 2 & 43.010 & 8 \\
\hline 15 & PRWCG & 27.243 & 17 & 36.927 & 18 \\
\hline 16 & PRWCG (5\% Nanopowder graphite) & 28.729 & 14 & 38.336 & 17 \\
\hline 17 & PRWCG (10\% Nanopowder graphite) & 28.223 & 16 & 39.233 & 14 \\
\hline 18 & PRWCG (20\% Nanopowder graphite) & 30.853 & 9 & 41.034 & 12 \\
\hline
\end{tabular}




\section{CONCLUSION}

Weighed normalized fuzzy logic method can be considered a suitable and efficient tool for solving a decision-making problem of selecting materials. Selecting the best candidates among all proper materials which are used for absorbing plates installed in electrostatic precipitators, which are so sensitive and need long life span in corrosive environments, is of high importance. In this method, the score and ranking of every alternative material are determined. This is a major merit in comparison with other methods which only determine material ranking level. Scoring can provide a better vision for a designer to consider similarities and differences among all possible alternative materials. In this investigation, both weighting and fuzzy logic methods are applied so as to select the best conductive composite. Due to putting a great deal of emphasis on designing aspect of material selection in the fuzzy logic method, fuzzy logic results are just shown; however, to increase the accuracy of the final choice in selecting suitable materials, it is better to consider both methods together, which helps to avoid possible faults. Finally, it is shown that both methods can be used together to provide a more efficient tool for selecting the best materials. In modified fuzzy logic method, based on weighing action carried out by designer and determination of bad, medium and good areas for given parameters, the best resin is vinyl ester and the best composite is VRWCG. The conventional weighing method has also provided similar ranking and the difference which can be seen between these two methods is due to the fact that there is slight difference among resins' indexes in the modified fuzzy method; therefore, if a given resin depicts larger number, assigned to the value of some parameters, the differences between the material index and other resins will be trivial. Furthermore, considering the condition which electro filters absorbing plates work on, this method obviously shows that VRWCG composite has got the highest ranking through conductive composites selected among several composite groups. This result is in utter conformity with weighed method results; however, among conductive composites whose characteristics are so similar, only VRWCG which has the highest ranking among other composites is placed on the same rank in both weighing and normalized fuzzy methods; other conductive composites are not ranked in the same way. Considering high accuracy and speed of modified fuzzy method, results drawn from it are much more reliable than other results obtained by other methods.

\section{References}

[1] Ashby, M. F., Brechet, Y. J. M., Cebon, D., \& Salvo, L. (2003). Selection Strategies for Materials and Processes. Materials and Design, 25(1), 51-67. https://doi.org/10.1016/S0261-3069(03)00159-6

[2] Ashby, M. F. (1992). Material Selection in Mechanical Design / Oxford Pergamon Press.

[3] Trethewey, K. R., Wood, R. J. K., Puget, Y., Roberge, P. R. (1998). Development of a Knowledge-Based System for Materials Management. Materials and Design, 19(1-2), 3956. https://doi.org/10.1016/S0261-3069(98)00010-7
[4] Amen, R. \& Vomacka, P. (2001). Case-Based Reasoning as a Tool for Materials Selection. Materials and Design, 22(5), 353-358. https://doi.org/10.1016/S0261-3069(00)00105-9

[5] Chen, J. L., Sun, S. H., Hwang, W. C. (1995). An Intelligent Data Base System for Composite Material Selection in Structural Design. Engineering Fracture Mechanics, 50(5-6), 935-946. https://doi.org/10.1016/0013-7944(94)E0068-R

[6] Sapuan, S. M. (2001). A Knowledge-Based System for Materials Selection in Mechanical Engineering Design. Materials and Design, 22(8), 687-695. https://doi.org/10.1016/S0261-3069(00)00108-4 Edwards, K. L. (2005). Selecting Materials For Optimum Use In Engineering Components. Materials and Design, 26(5), 469-473. https://doi.org/10.1016/j.matdes.2004.07.004

[7] Takuma, M., Shibasaka, T., Teshima, T., Iwai, Y., \& Honda, T. (1994). Study on Support System for Materials Selection in the Design Process. Trans JSME C, 60(574), 294-300. https://doi.org/10.1299/kikaic.60.2184

[8] Sarfaraz Khabbaz, R., Dehghan Manshadi, B., Abedian, A., \& Mahmudi, R. (2008). A Simplified Fuzzy Logic Approach for Materials Selection in Mechanical Engineering Design. Materials and Design, 30(3), 687-697. https://doi.org/10.1016/j.matdes.2008.05.026

[9] Liao, T. W. (1996). A Fuzzy Multicriteria Decision-Making Method For Material Selection. Journal of Manufacturing Systems, 15(1), 1-12. https://doi.org/10.1016/0278-6125(96)84211-7

[10] Shanian, A. \& Savadogo, O. (2006). A Material Selection Model Based On The Concept Of Multiple Attribute Decision Making. Materials and Design, 27(4), 329-337. https://doi.org/10.1016/j.matdes.2004.10.027

[11] Shanian, A. \& Savadogo, O. (2006). TOPSIS MultipleCriteria Decision Support Analysis for Material Selection of Metallic Bipolar Plates for Polymer Electrolyte Fuel Cell. Journal of Power Sources, 159(2), 1095-1104. https://doi.org/10.1016/j.jpowsour.2005.12.092

[12] Chen, R. W., Navin-Chandra, D., Kurfess, T., \& Prinz, F. B. (1994). A Systematic Methodology of Material Selection with Environmental Considerations. Proceedings of the IEEE international symposium on electronics and the environment / San Francisco, CA, USA, 252-257. https://doi.org/10.1109/ISEE.1994.337249

[13] Ribeiro, I., Pecas, P., Silva, A., \& Henriquez, E. (2008). Life Cycle Engineering Methodology Applied to Material Selection, a Fender Case Study. Journal of Cleaner Production, 16(17), 1887-99. https://doi.org/10.1016/j.jclepro.2008.01.002

[14] Bamkin, R. J. \& Piearcey B. J. (1990). Knowledge-Based Material Selection In Design. Materials and Design, 11(1), 25-29. https://doi.org/10.1016/0261-3069(90)90086-Y

[15] Ashby, M. F. (1989). Materials Selection in Conceptual Design. Materials Science and Technology, 5(6), 517-525. https://doi.org/10.1179/mst.1989.5.6.517

[16] Esawi, A. M. K. \& Ashby, M. F. (1996). Systematic Process Selection In Mechanical Design. Proceedings of the ASME design engineering technical conference / Irvine (CA), USA, 18-22.

[17] Ashby, M. F. (2000). Multi-Objective Optimization in Material Design and Selection. Acta Materialia, 48(1), 359369. https://doi.org/10.1016/\$1359-6454(99)00304-3

[18] Garton, D. A., Kang, K. J., Fleck, N. A., \& Ashby, M. F. (1992). Materials Selection for Minimum Weight Fatigue Design. Theory Concept Number Anal Fatigue, 359-376.

[19] Farag M., Materials Selection For Engineering Design. // Prentice Hall; (1997), pp. 227-234

[20] Manshadi, B. D., Mahmudi, H., Abedian, A., \& Mahmudi, R. (2007). A Novel Method for Materials Selection in Mechanical Design: Combination of Non-Linear 
Normalization and a Modified Digital Logic Method. Materials and Design, 28(1), 8-15.

https://doi.org/10.1016/j.matdes.2005.06.023

[21] Fayazbakhsh, K., Abedian, A., \& Dehghan Manshadi, B. (2009). Introducing a Novel Method for Materials Selection in Mechanical Design Using Z-Transformation in Statistics for Normalization of Material Properties. Materials and Design, 30(10), 4396-4404. https://doi.org/10.1016/j.matdes.2009.04.004

[22] Venkata Rao, R. (2008). A Decision Making Methodology for Material Selection Using an Improved Compromise Ranking Method. Materials and Design, 29(10), 19491954. https://doi.org/10.1016/j.matdes.2008.04.019

\section{Contact information:}

\section{Ali Akbar Lotfi NEYESTANAK}

Department of Engineering,

Yadegar-e-Imam Khomeini (RAH) Shahre Rey

Branch Islamic Azad University, Tehran, Iran

Postal address: 18155-144

E-mail: aklotfi@gmail.com

\section{Gasem ASADI}

Iranian oil pipeline and telecommunication company (IOPTC)

Qarani St, No. 135, Tehran Province, Tehran, Iran

\section{Saeed DANESHMAND}

Department of Mechanical Engineering

Majlesi Branch, Islamic Azad University, Isfahan, Iran

Postal address: 8631656451

E-mail: saeed_daneshmand@yahoo.com 